\title{
Le cadre institutionnel de lutte contre la corruption en RDC : analyse critique du cadre juridique de l'agence de prévention et de lutte contre la corruption
}

\author{
Par Clément Shamashanga Minga*
}

\section{Résumé}

Consacrée à l'examen du cadre juridique régissant l'Agence de Prévention et de Lutte contre la Corruption instituée par l'ordonnance n²0/013 bis du 17 mars 2020, l'étude démontre que ce cadre juridique présente un certain nombre des faiblesses qui entament la légitimité et la crédibilité de cette agence, hypothéquant ainsi les chances de réussite de sa mission. Elle indique que la création de l'APLC par une ordonnance, acte de l'exécutif, n'est pas de nature à assurer la pérennité et la stabilité de cette agence; le Président de la République pouvant à tout moment modifier ou abroger son acte comme bon lui semble. Aussi, l'étude révèle que l'ordonnance ne garantit pas l'indépendance et l'autonomie de l'agence qui est placée sous l'autorité directe du Président de la République. La dépendance de l'agence à l'égard du Président de la République conduit à la politisation de cette institution qui fait partie de services spécialisés du Cabinet du Chef de l'Etat.

L'étude révèle également que l'ordonnance créant l'APLC n'assure aucune protection aux membres et personnel de l'agence. Ceux-ci ne bénéficie d'aucun mandat; leur carrière dépend largement du Président de la République qui peut mettre fin à leurs fonctions à tout moment. Les animateurs et le personnel de l'agence ne bénéficient d'aucune protection en matière des poursuites pénales pour les actes commis dans l'exercice ou à l'occasion de l'exercice de leurs fonctions.

Elle suggère la mise en place d'une commission nationale de lutte contre la corruption réellement indépendante ayant statut d'une institution d'appui à la démocratie et dont les membres seront choisis parmi les personnalités indépendantes disposant d'une expérience avérée en matière de lutte contre la corruption.

\footnotetext{
Abstract

The study examines the legal framework governing the Agency for the Prevention and Fight against Corruption (APLC), established by Ordinance $n^{\circ} 20 / 013$ bis of March 17, 2020. It shows that the legal framework governing the APLC has a number of weaknesses that undermine the legitimacy and credibility of this agency, thus jeopardizing the chances

* Assistant à l'Institut Supérieur Pédagogique de Mweka (Kasaï), Avocat au Barreau de Matadi, Chercheur au Centre de Recherches et d'Etudes sur l'Etat de Droit en Afrique et Apprenant de troisième cycle en Droit public à l’Université de Kinshasa. Email : shamaclement@gmail.com.
} 
of success of its mission. It indicates that the creation of the APLC by an ordinance, an act of the executive, is not likely to ensure the durability and stability of this agency; the President of the Republic can at any time modify or repeal his act as he sees fit. The study also reveals that the ordinance does not guarantee the independence and autonomy of the agency, which is placed under the direct authority of the President of the Republic. The agency's dependence on the President of the Republic leads to the politicization of this institution, which is part of the specialized services of the Office of the Head of State.

The study also reveals that the ordinance creating the APLC does not provide any protection for the agency's members and staff. They have no mandate; their careers depend largely on the President of the Republic, who can terminate their functions at any time. The leaders and staff of the agency have no protection from criminal prosecution for acts committed in the exercise or on the occasion of the exercise of their functions.

It suggests the establishment of a truly independent national anti-corruption commission with the status of an institution supporting democracy, whose members will be chosen from among independent personalities with proven experience in the fight against corruption.

\section{Introduction}

La République Démocratique du Congo (RDC) comme la majorité des Etats africains est confrontée, depuis plusieurs années, à la corruption endémique à tous les échelons de la société. Cette corruption lui fait échapper des millions des dollars chaque année et empêche son développement.

Pour sortir de cette situation, elle s'est engagée résolument à lutter contre la corruption par la mise en place d'un dispositif juridique et institutionnel dont l'objectif vise l'éradication de ce cancer qui sape tous les efforts fournis par le Gouvernement et ses partenaires au développement. C'est dans ce sens qu'il faut situer la mise en place de l'Agence de prévention et de lutte contre la corruption qui fait l'objet de la présente étude.

Il s'ensuit que la mise en place de l'agence de prévention et de lutte contre la corruption doit être perçue, d'une part, comme la mise en œuvre par la République Démocratique du Congo des obligations internationales, régionales et sous régionales découlant des convention de lutte contre la corruption dont elle est partie et, d'autre part, comme l'expression de la volonté des nouvelles autorités de lutter contre la corruption qui est l'une des causes qui freinent le développement de ce pays.

\section{A. La mise en place de l'Agence comme la mise en ouvre des conventions internationales de lutte contre la corruption}

La mise en place de l'Agence de prévention et de lutte contre la corruption doit être regardée comme l'application par la RDC des dispositions des conventions internationales de lutte contre la corruption qu'elle a ratifiée. En effet, la Convention des Nations Unies contre 
la corruption, adoptée par l'Assemblée Générale dans sa Résolution 58/4 du 31octobre 2003, impose à chaque Etat partie l'obligation de mettre en place un ou plusieurs organes chargés de prévenir la corruption par des moyens tels que l'application des politiques visées à l'article 5 de ladite convention notamment la supervision et la coordination de la politique de lutte contre la corruption ainsi que la diffusion des connaissances concernant la prévention de la corruption ${ }^{1}$. L'article 36 de la même convention oblige à chaque Etat partie de mettre en place un ou plusieurs organes ou des personnes spécialisées dans la lutte contre la corruption par la détection et la répression.

La convention de l'Union Africaine sur la prévention et la lutte contre la corruption impose également, en son article 5 point 3 , à chaque Etat partie l'obligation notamment de mettre en place, de rendre opérationnelles et de renforcer des autorités ou agences nationales indépendantes chargées de lutter contre la corruption ${ }^{2}$. Le protocole de lutte contre la corruption signée le 14 aout 2001 par les Etats members de la Communauté de développement de l'Afrique australe impose aussi à chaque Etat partie l'obligation de mettre en place des institutions chargées de mettre en œuvre des mécanismes de prévention, de dépistage, de punition et d'éradication de la corruption ${ }^{3}$.

La RDC qui a signé et ratifié ces instruments juridiques internationaux de lutte contre la corruption est dans l'obligation de prendre les dispositions nécessaires pour appliquer ces conventions en instituant comme exigé un organe spécialisé chargé de prévenir et de lutter contre la corruption. Ainsi, la création de l'agence de lutte contre la corruption est à considérer comme un pas important dans la mise en œuvre en droit interne des conventions de lutte contre la corruption par la RDC.

Mais il faut dire que ce n'est pas pour la première fois que la RDC mette en place l'institution chargée de lutte contre la corruption. Plusieurs institutions ont été mises en place depuis le début de la transition en 2003 avec notamment la création de la commission nationale de lutte contre la corruption ${ }^{4}$, la Cellule de renseignements financiers ${ }^{5}$. Force est de reconnaitre que ces institutions dont certaines existent jusqu'à ce jour n'ont pas réalisés les résultats escomptés en matière de lutte contre la corruption notamment à cause

1 Lire l'Article 6 de la Convention des Nations Unies contre la corruption, in Réseau des Parlementaires Africains contre la corruption, Recueil des instruments juridiques de lutte contre la corruption en RDC, Paris, Editions Droit-Afrique, 2018, p.9.

2 Article 5 de la Convention de l'Union Africaine sur la prévention et la lutte contre la corruption, in Réseau des Parlementaires Africains contre la corruption, in Recueil des instruments juridiques de lutte contre la corruption en RDC, Op.Cit., p.51.

3 Article 4 point g du protocole de la Communauté de développement de l'Afrique Australe (SADEC) contre la corruption, Recueil des instruments juridiques de lutte contre la corruption en RDC, Op.Cit., p.83.

4 Décret $n^{\circ} 116 / 2002$ du 29 aout 2002 portant institution, attributions et composition d'une commission de lutte contre la corruption, la fraude et la contrebande ainsi que la contrefaçon de la monnaie et des marques, Journal officiel de la RDC, $44^{\text {ème }}$ année, $n^{\circ} 22$ du 15 novembre 2002, col.5.

5 Instituée par l'article 17 de la Loi nº4/016 du 19 juillet 2004 portant lutte contre le blanchiment des capitaux et le financement du terrorisme. 
de l'absence et de l'engagement des dirigeants d'œuvrer en faveur de la lutte contre la corruption. Aujourd'hui, cette volonté semble se manifester dans le chef des nouveaux détenteurs du pouvoir. Ce qui justifie amplement la création de l'agence de prévention et de lutte contre la corruption.

\section{B. L'institution de l'agence comme la manifestation de l'engagement des autorités congolaise de lutter contre la corruption}

Au-delà de la mise en œuvre des obligations issues des conventions de lutte contre la corruption, la création de l'agence doit également être percue comme manifestation de la volonté des nouveaux dirigeants de la RDC de s'attaquer à ce fléau qu'est la corruption qui ronge tous les secteurs de la vie nationale et qui fait perdre au Congo plusieurs milliards de dollars chaque année. En effet, à la suite des élections du 30 décembre 2018, la RDC a connu, pour la première fois de son histoire, une passation pacifique du pouvoir avec l'élection de Monsieur Félix Antoine Tshisekedi Tshilombo à la présidence de la République. Cette passation civilisée du pouvoir entre le Président sortant Monsieur Joseph Kabila et entrant Monsieur Félix Tshisekedi a constitué une bonne opportunité pour lutter contre la corruption.

Dès son élection, le Président Félix Tshisekedi a affirmé sa volonté et sa détermination à lutter contre la corruption. Dans son premier discours à l'occasion de la prestation de son serment constitutionnel, le nouveau Président de la République avait affirmé sa volonté de lutter contre la corruption : " Le Gouvernement que nous allons nommer prochainement et qui sera investi par le parlement va décliner son action politique sur plusieurs priorités

notamment une lutte efficace et déterminée contre la corruption et les antivaleurs.... ${ }^{6}$. Et d'ajouter que «nous allons initier une stratégie de lutte contre la corruption dans l'ensemble des rouages de l'administration publique. Un accent particulier sera porté sur la lutte contre la délinquance fiscale $»^{7}$. Le Président de la République a également réaffirmé son engagement à lutter contre la corruption à l'occasion de l'ouverture du séminaire organisé à Kinshasa en date du 13 mai 2019 en faveur des Gouverneurs et Vice-Gouverneurs des provinces.

Je combattrai, avec la dernière énergie, la corruption, le détournement des biens publics, le tribalisme, le népotisme, la concussion, le clientélisme, l'incivisme et j'en passe ${ }^{8}$.

6 Allocution du Président Félix Antoine TshisekediTshilombo à l'occasion de la prestation de son serment constitutionnel, Kinshasa, le 24 janvier 2019, Recueil des discours officiels, Direction de communication du Chef de l'Etat, janvier 2020, p.19.

7 Allocution du Président Félix Antoine TshisekediTshilombo à l'occasion de la prestation de son serment constitutionnel, note 6 .

8 Allocution du Président Félix Antoine TshisekediTshilombo à l'occasion de l'ouverture du séminaire des Gouverneurs et Vice- gouverneurs des provinces, Kinshasa, 13 mai 2019, Recueil des discours officiels, Direction de communication du Chef de l'Etat, janvier 2020, p.71. 
La lutte contre la corruption et les crimes économiques figure dans la vision du Président de la République et dans le programme du Gouvernement approuver par l'Assemblée nationale.

Il s'observe une réelle volonté des autorités congolaises de déclarer la guerre à la corruption et aux crimes économiques. Loin d'être un simple slogan ou une simple déclaration d'intention, l'engagement des autorités de lutter contre la corruption s'est traduit par certains actes encourageants. En effet, depuis l'avènement du Président Félix Tshisekedi au pouvoir, un certain nombre des hautes personnalités ont été poursuivies et condamnées soit pour détournement de deniers publics, soit pour corruption. L'on peut mentionner le cas de la condamnation de l'ancien Ministre de la santé et de l'ancien Président de l'Assemblée provinciale de la Ville de Kinshasa.

Les poursuites et les condamnations intervenues dans le cadre des procès dits « Procès de cent jours » sont révélatrices de la volonté des nouvelles autorités de lutter contre la corruption et les infractions y assimilées. En effet, les dénonciations des détournements des fonds publics alloués au programme de cent jours lancé par le Président de la République au début de son mandat ont conduit à l'inculpation et à la condamnation des plusieurs personnes dont le Directeur du Cabinet du Président de la République, Monsieur Vital Kamerhé. L'arrestation et la condamnation de Monsieur Vital Kamerhé et d'autres personnalités de haut rang ont été considérées comme un signal fort et un bond important dans la lutte contre la corruption et les détournements des deniers publics.

De ce qui précède, l'on peut dire que la mise en place de l'Agence répond à la volonté des dirigeants de la République Démocratique du Congo de lutter contre la corruption. Ceci dit, il nous revient maintenant de présenter le statut avant de se faire une idée sur sa mission et son organisation.

\section{Le statut de l'Agence de prévention et de lutte contre la corruption}

Le Statut de l'Agence est déterminé par l'article $1^{\text {er }}$ de l'ordonnance portant sa création, son organisation et son fonctionnement. Cet article dispose à son alinéa $1{ }^{\mathrm{er}}$ prévoit qu' " il est créé, au sein du Cabinet du Président de la République et sous son autorité, un service spécialisé dénommé Agence de prévention et de lutte contre la corruption ». Il ressort de cette disposition que l'Agence de prévention et de lutte contre la corruption est un service spécialisé du Président de la République. Elle fait partie de nombreux services qui constituent son Cabinet. L'Agence ne bénéficie pas de la personnalité juridique et de l'autonomie. Sa stabilité dépend de la volonté du Président de la République.

Cependant, le texte qui institute cette Agence indique qu'elle bénéficie de l'indépendance nécessaire pour l'accomplissement de sa mission. Ceci ressort des articles $1^{\text {er }}$ et 3 de l'ordonnance portant sa création, son organisation et son fonctionnement. L'article $1^{\text {er }}$ de cette ordonnance dispose à son alinéa 2 que « l'Agence formule ses politiques et exerce ses fonctions en toute indépendance dans les limites compatibles avec l'exercice de son mandat de lutte contre la corruption et les faits assimilés ». L'article 3 de la même 
ordonnance dispose que « dans l'accomplissement de sa mission, l'Agence dispose de l'indépendance nécessaire pour lui permettre d'exercer efficacement ses fonctions à l'abri de toute influence indue $»$.

Il ressort de la lecture de ces dispositions que l'Agence bénéficie de l'indépendance lui permettant d'exercer librement ses missions. Mais, le fait que l'Agence soit placée directement sous l'autorité du Président de la République, à qui elle rend compte de ses activités, amène à s'interroger sur la portée réelle de l'indépendance conférée à ce service spécialisé. Etant donné que l'Agence est placée sous l'autorité du Président de la République, il est clair que ce dernier exerce une influence réelle sur cette agence et que son Directeur de cabinet est le chef hiérarchique des dirigeants de l'Agence.

\section{Les Missions et attributions de l'Agence de prévention et de lutte contre la corruption}

L'agence a pour principale mission de définir et mettre en œuvre tous programmes permettant de détecter les agissement susceptibles d'être considérés comme relevant de la corruption ou d'une infraction y assimilée; de mener toutes études et diligenter des enquêtes nécessaires; de provoquer des poursuites pour faire sanctionner toutes personnes ou tous groupes de personnes, organisations, organismes, entreprises ou autres services impliqués dans les actes de corruption, de blanchiment des capitaux et de faits assimilés dans les conditions fixées par la réglementation en vigueur?

En rapport avec cette mission, l'Agence de prévention et de lutte contre la corruption a reçuplusieurs attributions. Premièrement, l'Agence a la compétence d'analyser, d'examiner et d'étudier tout indice, soupçon, acte, information ou rapport relatif à la corruption, au blanchiment des capitaux et/ou à des infractions assimilées qui serait porté à l'attention du Président de la République ou de ses services et de lui proposer des mesures appropriées de détection, de prévention et de sanction desdits comportements de manière plus efficace, des procédures de confiscation des produits illicites et de recouvrement des avoirs, revenus et autres profits obtenus au moyen de ces infractions, le tout dans le respect de la constitution et des lois de la République ${ }^{10}$.

Deuxièmement, l'Agence a compétence de mener des enquêtes sur les cas de corruption ou des infractions y assimilées. Dans ce cas, elle est autorisée de réunir et d'établir les preuves suffisantes de corruption et des actes assimilés, avec pouvoir d'entendre toute personne, de faire requérir la mise en cause de la personne ou entité concernée et, le cas échéant, son inculpation et des poursuites par les instances judiciaires compétentes,

9 Article 2 de $1^{\prime}$ Ordonnance $\mathrm{n}^{\circ} 20 / 013$ bis du 17 mars 2020 portant création, organisation et fonctionnement d'un service spécialisé dénommé « Agence de prévention et de lutte contre la corruption, Journal officiel de la République Démocratique du Congo, NSpécial du 18 avril 2020.

10 Article 2 point 1 de $1^{\prime}$ Ordonnance $n^{\circ} 20 / 013$ bis du 17 mars 2020 portant création, organisation et fonctionnement d'un service spécialisé dénommé « Agence de prévention et de lutte contre la corruption, note 9 . 
d'assurer que le dossier ainsi constitué et toutes ses pièces soient effectivement transmis et déposés auprès de l'organe judiciaire compétent et des poursuites effectivement engagées, et de veiller à ce que les conséquences de la corruption et des faits assimilés soient réparées, notamment par l'annulation ou la rescision d'un contrat, le retrait d'une concession ou de tout autre acte juridique analogue ou que soit prise toute autre mesure corrective ${ }^{11}$.

Troisièmement, l'Agence a compétencede prendre les dispositions appropriées pour assurer une protection efficace des témoins et des experts contre les représailles ou les actes d'intimidation dont ils feraient l'objet pour leur intervention tendant à caractériser les faits considérés; assurer la même protection à toute personne qui, de bonne foi et sur la base de soupçons raisonnables, signalera ou aura signalé à l'Agence des faits concernant les infractions ici visées; encourager les personnes ayant participé à la commission d'une telle infraction à coopérer avec l'Agence ${ }^{12}$.

Quatrièmement, l'Agence à la mission d'accompagner les entités ou personnes qui ont subi un préjudice du fait d'un acte de corruption, de blanchiment des capitaux ou d'une infraction y assimilée lorsqu'elles envisagent d'engager une action en justice pour en demander réparation à ceux dont la responsabilité sera établie ${ }^{13}$.

Cinquièmement, l'Agence a mandat de rechercher les opportunités d'accès à l'appui d'organismes internationaux afin de renforcer ses capacités d'enquêter et d'initier des poursuites pour mieux lutter contre la grande corruption en République Démocratique du Congo ${ }^{14}$.

Sixièmement, l'Agence a la compétence de collaborer avec les personnes, autorités, institutions et organisations de la société civile notamment qui, au niveau tant local qu'international, pourraient disposer d'éléments en rapport avec la corruption et les faits assimilés recueillis dans l'exercice de leurs propres attributions ${ }^{15}$.

11 Article 2 point 2 de l'Ordonnance $n^{\circ} 20 / 013$ bis du 17 mars 2020 portant création, organisation et fonctionnement d'un service spécialisé dénommé « Agence de prévention et de lutte contre la corruption, note 9 .

12 Article 2 point 3 de $1^{\prime}$ Ordonnance $n^{\circ} 20 / 013$ bis du 17 mars 2020 portant création, organisation et fonctionnement d'un service spécialisé dénommé « Agence de prévention et de lutte contre la corruption, note 9 .

13 Article 2 point 4 de l'Ordonnance $n^{\circ} 20 / 013$ bis du 17 mars 2020 portant création, organisation et fonctionnement d'un service spécialisé dénommé « Agence de prévention et de lutte contre la corruption, note 9 .

14 Article 2 point 5 de l'Ordonnance $n^{\circ} 20 / 013$ bis du 17 mars 2020 portant création, organisation et fonctionnement d'un service spécialisé dénommé « Agence de prévention et de lutte contre la corruption, note 9 .

15 Article 2 point 6 de l'Ordonnance $n^{\circ} 20 / 013$ bis du 17 mars 2020 portant création, organisation et fonctionnement d'un service spécialisé dénommé « Agence de prévention et de lutte contre la corruption, note 9 . 
Septièmement, l'Agence a la mission de concourir à l'élaboration et à la mise en œuvre effective des stratégies et mesures anticorruption à différents niveaux ${ }^{16}$.

Huitièmement, en fin, l'Agence a la charge de participer à la coordination administrative et centraliser puis diffuser les informations permettant d'aider à prévenir et à détecter les faits de corruption et des infractions y assimilées ${ }^{17}$.

Telles que présentées, les attributions de l'agence de prévention et de lutte contre la corruption peuvent être regroupées autour de quatre missions principales notamment la mission de prévention et de répression de la corruption ainsi que de coordination et de collaboration avec les organismes œuvrant dans le domaine de la lutte contre la corruption.

\section{La mission de prévention de la corruption}

La prévention de la corruption est la première mission d'une institution de lutte contre la corruption. Intervenant en amont, la mission de la prévention est la plus fondamentale de la politique criminelle de lutte contre la corruption ${ }^{18}$. Elle consiste à mettre en place des stratégies de sensibilisation des dirigeants et de la population sur les méfaits de la corruption $^{19}$.

La mission de prévention passe par l'éducation et la sensibilisation du public sur le phénomène de corruption ainsi que par la vulgarisation des instruments juridiques de lutte contre la corruption. La prévention de la corruption commande d'éduquer la population sur les dangers de la corruption et l'obligation qu'à chacun de la combattre.

Elle implique la diffusion des informations et des connaissances sur la corruption à l'intention du public et des groupes spécifiques ciblés en vue de susciter leur engagement et adhésion à la politique de lutte contre la corruption. En clair, la prévention de la corruption impose la mise en place des stratégies tendant à mobiliser les citoyens, la nécessité de lutter contre la corruption en mettant en place des programmes éducatifs et d'un système d'information et de communication sur la corruption ainsi que le renforcement des capacités opérationnelles des organismes publics et privés engagés dans la lutte contre la corruption.

Dans cet optique, l'ordonnance portant création de l'APLC attribue à cette agence au titre de la mission de prévention, la compétence de définir et de mettre en œuvre tous les programmes permettant de détecter les agissements susceptibles d'être considérés comme

16 Article 2 point 7 de 1'Ordonnance $n^{\circ} 20 / 013$ bis du 17 mars 2020 portant création, organisation et fonctionnement d'un service spécialisé dénommé « Agence de prévention et de lutte contre la corruption, note 9 .

17 Article 2 point 8 de l'Ordonnance $n^{\circ} 20 / 013$ bis du 17 mars 2020 portant création, organisation et fonctionnement d'un service spécialisé dénommé « Agence de prévention et de lutte contre la corruption, note 9 .

18 Daphné Latour et Pierre-Edouard Gondran de Robert, la lutte contre la corruption en France. 2013, une année décisive, Paris, EmeritPublishing, 2014, p.129.

19 Daphné Latour et Pierre-Edouard Gondran de Robert, la lutte contre la corruption en Frnce, note 20. 
relevant de la corruption ou d'une infraction y assimilée ${ }^{20}$. L'ordonnance créant l'APLC ne semble pas être très claire sur la détermination des activités rentrant dans la mission de prévention. Celles-ci sont à définir par l'APLC elle-même.

Dans ce sens, il sied de relever que l'APLC a, dans son plan stratégique national de prévention et de lutte contre la corruption ${ }^{21}$, décliné un certain nombre d'activités dans le cadre de sa mission de prévention de la corruption. Parmi les actions préventives de la corruption, il y a lieu de mentionner la sensibilisation des dirigeants et des citoyens au besoin de la transparence et de responsabiliser pour éviter la corruption et ses effets pervers $^{22}$; la mise en place d'un code de conduite obligatoire comprenant un chapelet des principes d'éthiques, de probité et d'équité capable d'entrainer la bonne gestion des affaires publiques $^{23}$; la mise en œuvre d'un dispositif d'alerte permettant aux agents ou experts de détecter les foyers et pratiques de corruption ${ }^{24}$.

Il convient de relever que si l'APLC a mis en place un plan stratégique national de lutte contre la corruption, ses actions en matière de sensibilisation et d'éducation des citoyens sur le phénomène de la corruption sont encore rares. Les quelques activités organisées par l'APLC dans le centre-ville de Kinshasa ont trait à la publication de la stratégie nationale de prévention et de lutte contre la corruption et de celle du code de bonne conduite de ses agents $^{25}$. Il y a lieu de signaler la participation de son Coordonnateur à l'émission populaire «parole aux auditeurs » organisée par la Radio Okapi.

L'ampleur de la corruption en RDC impose, pour le renversement de la tendance, une approche préventive réfléchie comprenant un certain nombre d'actions à impact visible alliant la sensibilisation à l'éducation de masses sur les effets de la corruption. L'organisation des séminaires, des ateliers et des conférences ainsi que des campagnes pourraient permettre d'atteindre un large public.

\section{La détectionde la corruption}

Il est admis que la lutte contre la corruption passe nécessairement par la répression des actes de corruption qui n'est rendue possible que dès lors que ces derniers ont été

20 Article 2 point 8 de l'Ordonnance $\mathrm{n}^{\circ} 20 / 013$ bis du 17 mars 2020 portant création, organisation et fonctionnement d'un service spécialisé dénommé « Agence de prévention et de lutte contre la corruption, note 9 .

21 Agence de prévention et de lutte contre la corruption, Stratégie nationale de lutte contre la corruption 2020-2030, Kinshasa, APLC, 2020.

22 Agence de prévention et de lutte contre la corruption, Stratégie nationale de lutte contre la corruption 2020-2030, note 21, p.15.

23 Agence de prévention et de lutte contre la corruption, Stratégie nationale de lutte contre la corruption 2020-2030, note 21, p.15.

24 Agence de prévention et de lutte contre la corruption, Stratégie nationale de lutte contre la corruption 2020-2030, note 21, p.16.

25 Agence de prévention et de lutte contre la corruption, Stratégie nationale de lutte contre la corruption 2020-2030, note 21, p.16. 
détectés $^{26}$. La détection est ainsi l'une des principales missions d'une institution de lutte contre la corruption. Cette mission passe par la recherche des actes de corruption afin de traduire leurs auteurs en justice.

L'ordonnance portant création de l'APLC assigne à cette derniere la mission de détecter la corruption. Dans le cadre de cette mission, l'APLC est chargée de mener les études et de diligenter des enquêtes, de provoquer des poursuites pour faire sanctionner toutes les personnes ou tous groupes des personnes, organisations, organismes, entreprises ou autres services impliqués dans les actes de corruption, de blanchiment des capitaux et des faits y assimilés ${ }^{27}$. L'APLC a, dans le cadre de la mission de détection de la corruption, la charge de conduire les enquêtes, réunir et d'établir les preuves suffisantes de corruption et des actes assimilés ainsi que d'entendre toute personne présumée auteur des acteurs de corruption, de faire requérir la mise en cause de personne ou entité impliquée dans la corruption et de le traduire devant les instances judiciaires ${ }^{28}$.

Dans l'exercice de sa mission de détection de la corruption, l'APLC est investi du pouvoir de se saisir d'office de tout acte ou fait de corruption ou de faits y assimilés dont elle a connaissance ${ }^{29}$. Elle peut également recevoir les réclamations, les plaintes et les dénonciations de n'importe quelle personne physique ou morale ${ }^{30}$. Cette mission autorise les membres de l'APLC d'entendre et de mettre en garde à vue les présumés auteurs d'actes de corruption ainsi que de dresser les procès-verbaux faisant foi. C'est pour cette raison que certains des agents de l'APLC sont dotés de la qualité d'officier de police judiciaire à compétence générale ${ }^{31}$.

Il sied de relever que l'APLC n'a pas le pouvoir de traduire directement devant les juridictions les présumés auteurs d'actes de corruption ou des actes y assimilés. Cette compétence revient au procureur général qui a le monopole de l'action public. Ce lien permet au magistrat du parquet de contrôler et d'endosser les actes posés par les membres

26 Daphné Latour et Pierre-Edouard Gondran de Robert, la lutte contre la corruption en France, note 20, p.140.

27 Article 2 de l'Ordonnance ${ }^{\circ} 20 / 013$ bis du 17 mars 2020 portant création, organisation et fonctionnement d'un service spécialisé dénommé « Agence de prévention et de lutte contre la corruption, note 9 .

28 Article 2 de l'Ordonnance ${ }^{\circ} 20 / 013$ bis du 17 mars 2020 portant création, organisation et fonctionnement d'un service spécialisé dénommé « Agence de prévention et de lutte contre la corruption, note 9 .

29 Article 3 de l'Ordonnance $n^{\circ} 20 / 013$ bis du 17 mars 2020 portant création, organisation et fonctionnement d'un service spécialisé dénommé « Agence de prévention et de lutte contre la corruption, note 9 .

30 Article 3 de l'Ordonnance $n^{\circ} 20 / 013$ bis du 17 mars 2020 portant création, organisation et fonctionnement d'un service spécialisé dénommé « Agence de prévention et de lutte contre la corruption, note 9 .

31 Article 11 de l'Ordonnance $\mathrm{n}^{\circ} 20 / 013$ bis du 17 mars 2020 portant création, organisation et fonctionnement d'un service spécialisé dénommé « Agence de prévention et de lutte contre la corruption, note 9 . 
de l'APLC dans l'exercice de leur mission de détection de la corruption. Ce qui permet également d'écarter l'argument selon les actes de l'APLC échappe à tout contrôle comme soutenu par une certain opinion.

S'il est vrai que du fait qu'il soit placée sous l'autorité du Président de la République, l'APLC échappe au contrôle politique du parlement, il est clair que les actes posés par cette agence n'échappe pas, en revanche, au contrôle des autorités judiciaires. Les officiers de police judiciaire étant placés sous l'autorité du procureur en RDC, il ne fait l'ombre d'aucun doute que les membres de l'APLC ayant la qualité d'officier de police judiciaire sont soumis à l'autorité du procureur de la République.

\section{La coordination des organismes nationaux de lutte contre la corruption}

Au-delà de sa mission de prévention et de détection de la corruption, l'APLC a également la mission de coordination des organismes œuvrant dans la lutte contre la corruption. Cette mission lui est expressément reconnue par l'article 3 de l'ordonnance qui la crée. Cet article dispose, en effet, que " l'Agence coordonne tous les services et organismes publics en charge de la lutte contre la corruption, le blanchiment des capitaux et les infractions y assimilés ». Cette disposition fait de l'APLC l'autorité nationale de référence en matière de lutte contre la corruption.

Il sied de relever qu'en matière de lutte contre la corruption, la RDC a mis en place plusieurs organismes notamment la Cellule Nationale de Renseignements Financiers (CENAREF), l'Observation de Surveillance de la Corruption et de l'Ethique Professionnelle (OSCEP), l'Inspection Générale des Finances (IGF), la Cour des comptes et la Coordination pour le Changement de Mentalité.

La mission de coordination permet l'harmonisation des politiques anti-corruption que les différents organes en charge de lutte contre la corruption sont appelés à mettre en œuvre.

\section{La coopération avec les organisations nationales et internationales de lutte contre la corruption}

L'APLC a, enfin, la mission de collaboration avec les organismes intervenant dans la lutte contre la corruption. A ce titre, elle est chargée de rechercher les opportunités d'accès à l'appui d'organismes internationaux afin de renforcer ses capacités d'enquêter et d'initier des poursuites pour mieux lutter contre la grande corruption en $\mathrm{RDC}^{32}$. L'APLC est chargée de collaborer avec les personnes, autorités, institutions et organisations de la société civile

32 Article 2 de l'Ordonnance ${ }^{\circ} 20 / 013$ bis du 17 mars 2020 portant création, organisation et fonctionnement d'un service spécialisé dénommé « Agence de prévention et de lutte contre la corruption, note 9 . 
notamment qui, au niveau tant local qu'international, pourraient disposer d'éléments en rapport avec les faits assimilés recueillis dans l'exercice de leurs propres attributions ${ }^{33}$.

Il sied de relever que la coopération avec les organismes et associations intervenant dans la lutte contre la corruption est une exigence d'efficacité pour la lutte contre la corruption. Cette coopération est même voulue par les conventions de lutte contre la corruption qui demandent aux Etats parties d'organiser la coopération entre les organes qui luttent contre la corruption. La coopération entre l'APLC avec les organisations de la société civile et les medias est nécessaire pour la lutte efficace contrela corruption.

\section{E. Organisation et fonctionnement de l'APLC}

Pour réussir sa mission, l'Agence est dotée de trois organes dont le Comité de pilotage, la Coordination et le Comité technique ${ }^{34}$.

\section{Le Comité de pilotage}

Le Comité de pilotage est considéré comme un organe d'orientation et de contrôle de l'Agence. Il est chargé d'assurer le respect strict de la lettre et de l'esprit de la mission confiée à l'Agence et de veiller à l'atteinte de ses objectifs ${ }^{35}$. C'est le Comité qui approuve le budget de l'agence et qui veille sur la mise en œuvre de ce budget.

Le Comité de pilotage est composé d'un délégué du Cabinet du Président de la République, d'un membre de la Coordination de l'agence, d'un délégué de chacun des ministères sectoriels dont celui de justice, budget, finances et droits humains, d'un délégué de la Coordination pour le changement de mentalités, d'un délégué de l'observation de surveillance de la corruption et de l'éthique professionnelle, d'un délégué de chacun des organismes en charge de la lutte contre le blanchiment des capitaux et de trois délégués de la société civile ${ }^{36}$.

33 Article 2de l'Ordonnance n²0/013 bis du 17 mars 2020 portant création, organisation et fonctionnement d'un service spécialisé dénommé « Agence de prévention et de lutte contre la corruption, note 9 .

34 Article 5 de l'Ordonnance n²0/013 bis du 17 mars 2020 portant création, organisation et fonctionnement d'un service spécialisé dénommé « Agence de prévention et de lutte contre la corruption, note 9 .

35 Article 6 de l'Ordonnance ${ }^{\circ} 20 / 013$ bis du 17 mars 2020 portant création, organisation et fonctionnement d'un service spécialisé dénommé « Agence de prévention et de lutte contre la corruption, note 9 .

36 Article 7 de l'Ordonnance $n^{\circ} 20 / 013$ bis du 17 mars 2020 portant création, organisation et fonctionnement d'un service spécialisé dénommé « Agence de prévention et de lutte contre la corruption, note 9 . 
Il est prévu la possibilité pour le Président de la République de faire participer au comité de pilotage les partenaires au développement qui apportent leur concoursà la mise en œuvre de la missionde l'agence ${ }^{37}$.

Il sied de souligner que le Comité de pilotage, dont les modalités de fonctionnement sont à déterminer par le règlement intérieur, est convoqué et présidé par le Président de la République ou son délégué38.

\section{La Coordination}

La Coordination est l'organe de gestion quotidienne de l'Agence. Elle a la charge d'exécuter les missions confiées à l'Agence, de préparer les dossiers traités avant de les transmettre aux autorités judiciaires compétentes pour les suites appropriées ${ }^{39}$. La Coordination a également la charge de rédiger et de soumettre au Président de la République des rapports circonstanciels, trimestriels et annuels sur l'ensemble de ses activités ${ }^{40}$. Elle a l'obligation de rendre public le résumé du rapport annuel de ses activités afin de permettre à l'opinion publique d'être informée sur l'avancement de la lutte contre la corruption en République Démocratique du Congo.

La Coordination est dirigée par un Coordonnateur qui est assisté de trois adjoints. Le Coordonnateur et ses adjoints sont nommés par le Président de la République lequel est compétent pour mettre fin à leurs fonctions ${ }^{41}$. Il est prévu que le coordonnateur et ses adjoints soient intègres, de bonne moralité et ne soient jamais impliqués dans les actes de corruption.

Il sied de relever que c'est le Coordonnateur qui assure la direction, organise et supervise l'ensemble des activités de l'agence et qui rend compte des activités de la Coordination directement au Président de la République à travers des notes, des avis et des rapports ${ }^{42}$.

37 Article 7 de l'Ordonnance $n^{\circ} 20 / 013$ bis du 17 mars 2020 portant création, organisation et fonctionnement d'un service spécialisé dénommé « Agence de prévention et de lutte contre la corruption, note 9.

38 Article 7 de l'Ordonnance ${ }^{\circ} 20 / 013$ bis du 17 mars 2020 portant création, organisation et fonctionnement d'un service spécialisé dénommé « Agence de prévention et de lutte contre la corruption, note 9 .

39 Article 8 de l'Ordonnance $n^{\circ} 20 / 013$ bis du 17 mars 2020 portant création, organisation et fonctionnement d'un service spécialisé dénommé « Agence de prévention et de lutte contre la corruption, note 9 .

40 Article 8 de l'Ordonnance $n^{\circ} 20 / 013$ bis du 17 mars 2020 portant création, organisation et fonctionnement d'un service spécialisé dénommé « Agence de prévention et de lutte contre la corruption, note 9 .

41 Article 8 de l'Ordonnance $n^{\circ} 20 / 013$ bis du 17 mars 2020 portant création, organisation et fonctionnement d'un service spécialisé dénommé « Agence de prévention et de lutte contre la corruption, note 9 .

42 Article 9 de l'Ordonnance $n^{\circ} 20 / 013$ bis du 17 mars 2020 portant création, organisation et fonctionnement d'un service spécialisé dénommé « Agence de prévention et de lutte contre la corruption, note 9 . 
C'est le Coordonnateur qui représente l'agence dans ses rapports avec les tiers et qui veille à ce que chaque membre de l'agence agisse en toute indépendance et impartialité dans l'exercice de ses attributions, notamment en ce qui concerne ses constatations, ses enquêtes et les conclusions de ses rapports ${ }^{43}$. C'est également lui qui exerce le pouvoir disciplinaire sur l'ensemble des membres de l'agence, à l'exception de ceux de la Coordination ${ }^{44}$.

Le Coordonnateur a rang de Conseiller spécial du Chef de l'Etat et est soumis au même régime administratif et rémunératoire, au même régime de déontologie et de discipline que le conseil spécial du Président de la République ${ }^{45}$. Il est aussi l'ordonnateur des dépenses de l'agence, et ce, dans la limite des crédits budgétaires et dans le respect de la règlementation budgétaire ${ }^{46}$. Il dispose d'un bureau composé d'un assistant, d'un secrétaire particulier, d'un chauffeur et de deux gardes du corps ${ }^{47}$.

Les Coordonnateurs adjoints, qui ont rang de conseillers principaux du Chef de l'Etat ${ }^{48}$, assistent le Coordonnateur et assument son intérim en cas d'absence ou d'empêchement, et ce, par ordre de préséance sur l'acte de nomination ${ }^{49}$. Le premier Coordonnateur adjoint est chargé de la détection, de la prévention et de l'administration; le deuxième s'occupe des plaintes, des enquêtes et investigations; et le troisième, quant à lui, est chargé des poursuites

43 Article 9 de l'Ordonnance $n^{\circ} 20 / 013$ bis du 17 mars 2020 portant création, organisation et fonctionnement d'un service spécialisé dénommé « Agence de prévention et de lutte contre la corruption, note 9 .

44 Article 9 de l'Ordonnance ${ }^{\circ} 20 / 013$ bis du 17 mars 2020 portant création, organisation et fonctionnement d'un service spécialisé dénommé « Agence de prévention et de lutte contre la corruption, note 9 .

45 Article 9 de l'Ordonnance ${ }^{\circ} 20 / 013$ bis du 17 mars 2020 portant création, organisation et fonctionnement d'un service spécialisé dénommé « Agence de prévention et de lutte contre la corruption, note 9 .

46 Article 9 de l'Ordonnance $n^{\circ} 20 / 013$ bis du 17 mars 2020 portant création, organisation et fonctionnement d'un service spécialisé dénommé « Agence de prévention et de lutte contre la corruption, note 9 .

47 Article 9 de l'Ordonnance n²0/013 bis du 17 mars 2020 portant création, organisation et fonctionnement d'un service spécialisé dénommé « Agence de prévention et de lutte contre la corruption, note 9 .

48 Article 10 de l'Ordonnance $\mathrm{n}^{\circ} 20 / 013$ bis du 17 mars 2020 portant création, organisation et fonctionnement d'un service spécialisé dénommé « Agence de prévention et de lutte contre la corruption, note 9 .

49 Article 10 de l'Ordonnance $n^{\circ} 20 / 013$ bis du 17 mars 2020 portant création, organisation et fonctionnement d'un service spécialisé dénommé « Agence de prévention et de lutte contre la corruption, note 9 . 
et du suivi des affaires fixées devant la justice ${ }^{50}$. Les Coordonnateurs adjoints disposent chacun d'un bureau composé d'un assistant, d'un chauffeur et de deux gardes du corps ${ }^{51}$.

\section{Le Comité technique}

Le Comité technique est l'organe technique à l'appui de l'action de la Coordination. Il est chargé notamment d'analyser, examiner et étudier dans les détails les actes, faits, informations ou rapports relatifs à la corruption et aux faits y assimilés qui portés à la connaissance de l'agence; de mener les investigations y afférentes; de réunir toutes les preuves et d'émettre des avis et recommandations à l'attention de la Coordination ${ }^{52}$. Le Comité technique est composé d'un groupe d'experts permanents et multisectoriels sélectionnés par le Coordonnateur et désignés par décision du Directeur de Cabinet du Président de la République, après approbation du Président de la République ${ }^{53}$. La Coordination peut inviter toute personne ou structure dont l'expertise s'avère nécessaire aux travaux du Comité technique. Il sied de noter que certains membres du Comité technique sont dotés de la qualité d'officier de police judiciaire ${ }^{54}$.

Les membres du Comité technique ont droit à un régime de rémunération et avantages correspondant à celui des conseillers au Cabinet du Président de la République.

\section{Du service du personnel d'appoint et des ressources}

Pour son fonctionnement, l'agence bénéficie, d'une part, d'un service du personnel d'appoint qui est constitué d'un personnel administratif et technique notamment, trois assistants, un secrétaire administratif, deux opérateurs de saisie, un agent de courrier, un agent protocole et un chauffeur; et d'autre part, d'une dotation émargeant du budget de l'Etat ainsi que de tout soutien et financement des partenaires et organismes intéressés à sa mission.

Comme l'on peut s'en rendre compte l'organisation de l'APLC est centralisée. L'agence ne dispose pas des représentations ni en province ni dans les entités territoriales

50 Article 10 de l'Ordonnance $n^{\circ} 20 / 013$ bis du 17 mars 2020 portant création, organisation et fonctionnement d'un service spécialisé dénommé « Agence de prévention et de lutte contre la corruption, note 9 .

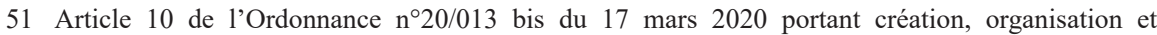
fonctionnement d'un service spécialisé dénommé « Agence de prévention et de lutte contre la corruption, note 9 .

52 Article 11 de l'Ordonnance $\mathrm{n}^{\circ} 20 / 013$ bis du 17 mars 2020 portant création, organisation et fonctionnement d'un service spécialisé dénommé «Agence de prévention et de lutte contre la corruption, note 9 .

53 Article 12 de l'Ordonnance $\mathrm{n}^{\circ} 20 / 013$ bis du 17 mars 2020 portant création, organisation et fonctionnement d'un service spécialisé dénommé «Agence de prévention et de lutte contre la corruption, note 9 .

54 Article 12 de l'Ordonnance $\mathrm{n}^{\circ} 20 / 013$ bis du 17 mars 2020 portant création, organisation et fonctionnement d'un service spécialisé dénommé « Agence de prévention et de lutte contre la corruption, note 9 . 
locales. Une telle structuration ne pourrait permettre à l'agence de mener avec efficacité son action sur toute l'étendue de la RDC. La lutte contre la corruption doit concerner l'ensemble du territoire national.

La présentation de l'organisation de l'APLC nous amène à apprécier objectivement le cadre juridique qui la régi.

\section{F. Appréciation critique du cadre juridique régissant l'APLC}

L'APLC est régie comme relevé plus haut par l'ordonnance n²0/013 bis du 17 mars 2020 qui la crée. Ce texte présente un certain nombre des faiblesses qui pourraient impacter négativement sur la mission de cette agence. D'abord, le statut de l'APLC tel que fixé par cette ordonnance est source d'inquiétude. Ensuite, l'ordonnance précitée ne garantit pas l'indépendance de l'Agence et ne protège pas le personnel de cette agence. Ce qui impacte également sur l'intégrité de ce personnel.

\section{Le statut de l'Agence tel que fixé par l'ordonnance est peu rassurant}

Le statut juridique de l'Agence de prévention et de lutte contre la corruption tel que fixé par l'ordonnance qui crée ce service soulève un certain nombre d'inquiétudes. En effet, l'Agence, comme nous l'avons démontré plus haut, n'est pas une autorité administrative indépendante. Elle n'est pas dotée de la personnalité et d'une autonomie tant sur le plan juridique que administratif et financier. Cette situation est déplorable à plusieurs égards. D'abord, le fait que l'agence soit mise en place par une ordonnance du Président de la République est une source d'instabilité dans la mesure où cet acte de l'exécutif peut être modifié à tout moment et en cas de changement du régime être abrogé par le nouveau dirigeant. L'on a vu le nouveau Président de la République Félix Antoine Tshisekedi abroger l'ordonnance de son prédécesseur nommant un conseiller spécial en charge de lutte contre la corruption et le blanchiment des capitaux. La création de l'agence par un acte de l'exécutif n'est pas adéquate.

Pour garantir la stabilité de l'agence, il aurait fallu qu'elle soit créée par une loi organique ou par une loi ordinaire. L'autorité attachée à la loi devrait conférer une légitimité et une autorité indéniable à l'agence ${ }^{55}$. Le souci d'assurer la pérennité de l'institution de lutte contre la corruption impose qu'elle soit créée par une loi pour éviter que son mandat ou sa mission ne soit modifiée à tout moment par le pouvoir exécutif dont les animateurs sont cités parmi les personnes corrompues.Faut-il rappeler que pour assurer la permanence des agences de lutte contre la corruption, les principes contenus dans le communiqué de Jakarta suggèrent qu'elles soient mise en place par un cadre juridique adéquat et stable comme la constitution ou une loi spéciale pour garantir leur continuité.

55 Gilles Badet, Luc Damiba, Stéphane B. Engueléguélé, Emmanuel Gaima, Chijioke K. Iwuamadi, SemouNdiaye et Shine Williams, Effectivité des agences nationales anti-corruption en Afrique de l'ouest, New York, 2016, p.250. 
Ensuite, l'agence ne dispose pas de l'autonomie juridique lui permettant de fixer son fonctionnement interne. Son règlement intérieur est arrêté par une décision du Directeur du cabinet du Chef de l'Etat. L'agence n'a pas non plus le pouvoir de recruter directement son personnel. Celui-ci est recruté par le Directeur de cabinet du Président de la République après approbation du Président de la République. Cette situation n'augure pas une bonne administration.

Enfin, l'agence ne dispose pas d'une autonomie financière. Ses ressources sont comprises dans celles allouées au Président de la République. Les dons et legs en faveur de l'agence doivent nécessairement passés par la présidence de la République.

\section{La dépendance de l'agence à l'égard du Président de la République et de son directeur de cabinet}

La lecture de l'ordonnance créant l'APLC révèle que l'agence est dépendante à l'égard du Président de la République. Ceci ressort de l'article $1^{\text {er }}$ de l'ordonnance portant création de l'agence qui fait de l'agence un service spécialisé au sein du Cabinet du Président et placé sous l'autorité de ce dernier. On le sait, conformément aux dispositions de l'article 5 de l'ordonnance $n^{\circ} 09 / 003$ du 30 janvier 2009 portant organisation et fonctionnement du Cabinet du Président de la République, c'est le Directeur de Cabinet du Chef de l'Etat qui assure la direction, l'organisation, la coordination et la surveillance de l'ensemble du Cabinet et qui exerce le pouvoir disciplinaire sur tous les membres du Cabinet. Au regard de cette disposition, il ne fait l'ombre d'aucun doute que le Directeur de Cabinet exerce également une influence sur l'agence de prévention et de lutte contre la corruption qui est placée sous l'autorité du Président de la République.

S'il est vrai que le fait que l'agence soit placée sous l'autorité du Président de la République lui place dans une position lui permettant d'exercer correctement sa mission en raison de la position qu'occupe le Chef de l'Etat au sein du système politique congolais, il est également vrai que cette position peut conduire à sa politisation et à son inféodation. Une telle situation est contraire aux dispositions des conventions internationales de lutte contre la corruption dont la RDC est partie.

Pour mémoire, les articles 6 et 36 de la convention des Nations Unies contre la corruption obligent chaque Etat partie d'accorder à l'organe ou aux organes de prévention de la corruption l'indépendance nécessaire, conformément aux principes fondamentaux de son système juridique, pour leur permettre d'exercer efficacement leurs missions à l'abri de toute influence indue. L'article 20 de la convention de l'Union Africaine sur la prévention et la lutte contre la corruption demande également à chaque Etat partie de faire en sorte que l'agence de lutte contre la corruption puisse jouir de l'indépendance et l'autonomie nécessaire pour exercer efficacement leurs fonctions. Cette obligation est encore reprise dans le communiqué de Jakarta sur les principes pour les institutions de lutte contre la corruption. 
Il nous revient qu'au regard de ces dispositions pertinentes des conventions internationales de lutte contre la corruption que la dépendance de l'Agence de prévention et de lutte contre la corruption à l'égard du Président de la République n'est pas conforme aux normes et pratiques internationales qui gouvernent les institutions de lutte contre la corruption. L'on le sait aucune institution de lutte contre la corruption ne peut être efficace si n'est réellement libre de toute influence de la part du pouvoir exécutif. Or, l'APLC est, de part son statut, soumis à l'autorité du Président de la République et de son directeur de Cabinet qui est le coordonnateur de tous les services qui dépendent de la présidence de la République.

\section{Le statut du personnel peu protecteur}

Le statut du personnel de l'APLC est également peu rassurant dans la mesure où il n'assure aucune protection aux animateurs de l'agence et à leurs subordonnés. D'abord, les animateurs de l'agence n'ont pas un mandat. Ils ont le statut du personnel politique œuvrant au sein du Cabinet du Président de la République à qui ils doivent leurs mandats. Le maintien ou non en fonction des membres de l'agence dépend entièrement du Président de la République et de sa volonté. D’ailleurs, le Président de la République a mis fin aux fonctions du Premier Coordonnateur de l'agence de son propre gré même si l'on sait que depuis son arrestation par le Procureur Général près la Cour d'Appel de Kinshasa/Gombe, plusieurs voix s'étaient levées pour exiger la démission du Coordonnateur de l'APLC.

Aussi, les agents œuvrant au sein du comité technique de l'APLC n'ont pas de contrat $\mathrm{du}$ travail. Ils sont également régis par le statut applicable au personnel œuvrant au cabinet du Président de la République. Les animateurs de l'agence et les membres du comité technique de cette dernière sont placés sous l'autorité du Directeur de cabinet du Chef de l'Etat.

En plus, les animateurs de l'agence et le personnel de cette dernière ne bénéficient d'aucune protection en matière des poursuites pénales de telle sorte qu'ils peuvent être arrêtés à tout moment par n'importe quelle autorité judiciaire comme il en a été le cas pour le Coordonnateur de l'agence qui avait été placé sous mandat d'arrêt provisoire par le Procureur Général près la Cour d'appel de Kinshasa-Gombe.

Un tel statut n'est pas de nature à permettre aux animateurs et personnel de l'agence d'exercer leurs activités sans craindre les représailles. Pareil statut est contraire au minima exigé par les conventions internationales de lutte contre la corruption ratifiées par la RDC ainsi que par les principes régissant les institutions de lutte contre la Corruption tels que repris dans le communiqué de Jakarata.

Selon ces principes, les dirigeants d'une institution de lutte contre la corruption doiventêtre désignés à l'issue d'un processus qui garantit leur apolitisme, impartialité, neutralité, intégrité et leur compétence. Ces principes suggèrent également que les animateurs d'une institution de lutte contre la corruption soient inamovibles et ne puissentêtre 
révoqués que par le biais d'une procédure légalement établie correspondant à la procédure de révocation de haute autorité indépendante expressément protégée par la loi.

Les mêmes principes suggèrent encore que les dirigeants et personnel d'une institution de lutte contre la corruption puissent bénéficier des immunités pour les protéger contre les poursuites pour tout acte commis dans le cadre de l'exercice de leurs missions.

Il sied de relever que la protectiondes membres d'une institution de lutte contre la corruption constitue une garantie supplémentaire de leur indépendance et une condition d'efficacité de l'institution.

\section{Le mode de recrutement des membres de l'Agence impact négativement sur leur intégrité}

Les conditions de recrutement des membres de l'APLC ne sont pas de nature à garantir leur intégrité ni leur indépendance. Bien que l'ordonnance portant création de l'APLC précise en son article 8 que le Coordonnateur et les Coordonnateurs adjoints de cette agence doivent êtreintègres, de bonne moralité et n'avoir jamais été impliqués dans les actes de corruption, il faut, cependant, relever qu'aucune condition en rapport avec la formation et l'expérience professionnelle n'est exigée. Le recrutement du coordonnateur et de ses adjoints dépend largement de la volonté du Président de la République qui, dans plupart de cas, choisi ses hommes de confiance parfois sans expérience dans le domaine de la lutte contre la corruption.

De même, le recrutement des experts permanents regroupés au sein du Comité Technique par le Coordonnateur de l'APLC ne répond à aucun critère de transparence ni à aucune condition de compétence et d'expérience. Le recrutement des agents de l'APLC semble être fondé sur les critères subjectifs qu'objectifs. Pareil recrutement risque d'impacter negativement sur l'intégrité des membres de l'agence. Les agissements des agents de l'APLC dans les enquêtes diligentées auprès d'ACCESS BANK en décembre 2020 ont mis au grand jour l'absence d'intégrité dans le chef des agents de cette agence.

En effet, en date du 10 décembre 2020, les agents de l'APLC qui menait une enquête sur les faits de blanchiment et de financement du terrorisme dans le chef de l'ACCESS BANK RDC dirigée par Monsieur ARINZE de nationalité nigériane se sont rendus nuitamment au siège de cette Banque pour exiger le payement d'une somme de 30000 dollars au titre de caution; somme qu'ils avaient reçue en espèce ${ }^{56}$. La scène a été filmée et diffusée sur les réseaux sociaux. Elle a provoqué un tollé et des réactions diverses dans l'opinion. Dans un communiqué rendu public le 15 décembre 2020, l'Association Congolaise d'Accès à la Justice, ACAJ, a considéré que les actes posés par les agents de l'APLC constituaient à l'égard de la banque un harcèlement commis dans le seul but de prendre l'argent du Directeur Général de l'ACCESS BANK, en violation des lois de la République.

56 Stanis Bujakera Tshiamala, «RDC : l'agence anticorruption de Tshisekedi accusée d'extorsion par Access Bank »,www.jeuneafrique.com/1092342/politique/rdc-lagence-anticorruption-de-tshiseked i-accusee-dextorsion-par-access-bank, (consulté le 15 février 2021). 
$\mathrm{Au}$ lendemain de cette scène, les avocats de la banque avait porté plainte contre les agents de l'APLC au Parquet Général près la Cour d'Appel de Kinshasa/Gombe. Cette plainte a abouti à la mise en détention du Coordonnateur de l'agence ${ }^{57}$. A la suite de cette arrestation, plusieurs voix s'étaient levées pour exiger la démission du Coordonnateur de l'APLC. Mais ce dernier est resté dans ses fonctions jusqu'à la nomination d'un nouveau Coordonnateur par le Président de la République en mai 2021.

Le comportement des agents de l'APLC est en contradiction avec les règles éthiques qui caractérisent le personnel d'une institution de lutte contre la corruption. En effet, appelés à lutter contre la corruption, les animateurs et les agents de l'agence doivent avoir une conduite exemplaire et être d'une bonne moralité ainsi que capable de résister à toute tentation de la corruption. C'est ainsi qu'il est suggéré que les animateurs et les agents d'une institution de lutte contre la corruption soient recrutés parmi les personnalités indépendantes disposant des qualités morales irréprochables. Il est recommandé aussi la mise en place d'un code de bonne conduite qui oblige les animateurs de l'agence de faire preuve d'une éthique exemplaire. Ces exigences n'ont malheureusement été prises par le Président de la République tant dans son ordonnance que le choix des animateurs de l'APLC.

L'examen du cadre juridique régissant l'APLC a démontré que l'ordonnance portant création de cette agence présente un certain nombre des faiblesses qui impactent négativement sur l'efficacité de l'agence. La résolution de cette situation passe par la mise en place d'une institution de lutte contre la corruption réellement indépendante.

\section{G. De la nécessité de mettre en place une Commission nationale de lutte contre la corruption en RDC}

En RDC, le souci de lutter contre la corruption qui freine le développement du pays est partagé par les autorités actuelles et par l'ensemble de la population. Cependant, une telle lutte ne pourrait devenir une réalité et produire des résultats escomptés sans la mise en place d'une véritable institution de lutte contre la corruption répondant aux normes et pratiques en la matière notamment aux dispositions pertinentes des conventions internationales de lutte contre la corruption ratifiées par la RDC.

Dans cet optique, la RDC doit mettre en place une Commission nationale de lutte contre la corruption. Cette Commission doit être instituée par une loi organique conformément à l'article 222 de la constitution et être dotée du statut d'une institution d'appui à la démocratie à l'instar de la Commission nationale des droits de l'homme. Elle devra être dotée d'une indépendance et d'une autonomie réelle. Ses membres doivent provenir des

57 Siméon ISAKO, «Affaire Access Bank : Gislain KIKANGALA, Coordonnateur de l'agence anticorruption placée en détention préventive », Affaire Access Bank : Gislain Kikangala, coordonateur de l'agence anti-corruption placé en détention préventive - CAS-INFO.CA, (consulté le 15 février 2021). 
différentes couches de la population et être choisis parmi les personnalités indépendantes disposant d'une expérience avérée dans le domaine de la lutte contre la corruption.

Instituée par une loi organique et dotée du statut d'une institution d'appui à la démocratie, la Commission nationale de lutte contre la corruption pourrait bénéficier d'une légitimité et d'un soutien de tous les acteurs politiques et sociaux ainsi que des partenaires de la RDC.

\section{Conclusion}

La présente étude a examiné le cadre juridique régissant l'Agence de Prévention et de Lutte contre la Corruption instituée par l'ordonnance n²0/013 bis du 17 mars 2020. Elle démontre que le cadre juridique régissant l'APLC présente un certain nombre des faiblesses qui entament la légitimité et la crédibilité de cette agence, hypothéquant ainsi les chances de réussite de sa mission.

Premièrement, la création de l'APLC par une ordonnance, acte de l'exécutif, n'est pas de nature à assurer la pérennité et la stabilité de cette agence; le Président de la République pouvant à tout moment modifier ou abroger son acte comme bon lui semble. Deuxièmement, l'ordonnance ne garantit pas l'indépendance et l'autonomie de l'agence qui est placée sous l'autorité directe du Président de la République. La dépendance de l'agence à l'égard du Président de la République conduit à la politisation de cette institution qui fait partie de services spécialisés du Cabinet du Chef de l'Etat.

Troisièmement, l'ordonnance n'assure aucune protection aux membres et personnel de l'agence. Ceux-ci ne bénéficie d'aucun mandat; leur carrière dépend largement du Président de la République qui peut mettre fin à leurs fonctions à tout moment. Aussi, les animateurs et le personnel de l'agence ne bénéficie d'aucune protection en matière des poursuites pénales pour les actes commis dans l'exercice ou à l'occasion de l'exercice de leurs fonctions.

L'étude révèle que le statut de l'APLC tel que fixé par l'ordonnance précitée n'est pas conforme aux dispositions des conventions internationales, régionales et sous-régionales ratifiées par la RDC en ce qu'il ne garantit pas l'indépendance et l'autonomie à l'agence.

Dans le but de se conformer aux normes et pratiques internationales régissant les institutions de lutte contre la corruption, l'étude propose la mise en place par la RDC d'une véritable commission nationale de lutte contre la corruption dotée d'une réelle indépendance et autonomie. Cette commission doit être instituée par une loi organique et être dotée du statut d'une institution d'appui à la démocratie. Ses membres doivent également provenir des différentes couches de la population. C'est à cette condition que son action pourrait bénéficier du soutien de la population et des partenaires intéressés par la lutte contre la corruption. 


\section{Bibliographie sommaire}

1. Convention des Nations Unies contre la corruption, in Réseau des Parlementaires Africains contre la corruption, Recueil des instruments juridiques de lutte contre la corruption en RDC, Paris, Editions Droit-Afrique, 2018.

2. Convention de l'Union Africaine sur la prévention et la lutte contre la corruption, in Réseau des Parlementaires Africains contre la corruption, Recueil des instruments juridiques de lutte contre la corruption en RDC, Paris, Editions Droit-Afrique, 2018.

3. Protocole de la Communauté de Développement de l'Afrique Australe (SADEC) contre la corruption, in Réseau des Parlementaires Africains contre la corruption, Recueil des instruments juridiques de lutte contre la corruption en RDC, Paris, Editions Droit-Afrique, 2018.

4. Décret $n^{\circ} 116 / 2002$ du 29 aout 2002 portant institution, attributions et composition d'une commission de lutte contre la corruption, la fraude et la contrebande ainsi que la contrefaçon de la monnaie et des marques, Journal officiel de la RDC, 44 ème année, $\mathrm{n}^{\circ} 22$ du 15 novembre 2002, col.5.

5. Loi ${ }^{\circ} 04 / 016$ du 19 juillet 2004 portant lutte contre le blanchiment des capitaux et le financement du terrorisme.

6. Présidence de la République, Recueil des discours officiels, Direction de communication du Chef de l'Etat, janvier 2020.

7. Ordonnance ${ }^{\circ} 20 / 013$ bis du 17 mars 2020 portant création, organisation et fonctionnement d'un service spécialisé dénommé « Agence de prévention et de lutte contre la corruption, Journal officiel de la République Démocratique du Congo, №Spécial du 18 avril 2020.

8. Daphné Latour et Pierre-Edouard Gondran de Robert, la lutte contre la corruption en Frnce. 2013, une année décisive, Paris, EmeritPublishing, 2014.

9. Gilles Badet, Luc Damiba, Stéphane B. Engueléguélé, Emmanuel Gaima, Chijioke K. Iwuamadi, SemouNdiaye et Shine Williams, Effectivité des agences nationales anti-corruption en Afrique de l'ouest, New York, 2016.

10. Réseau de Parlementaires Africains contre la Corruption, Recueil des instruments juridiques de lutte contre la corruption en RDC, Paris, Editions Droit-Afrique, 2018.

11. Agence de prévention et de lutte contre la corruption, Stratégie nationale de lutte contre la corruption 2020-2030, Kinshasa, APLC, 2020. 